ORIGINAL ARTICLE

\title{
Insulin-like growth factor I correlates with lean body mass in cystic fibrosis patients
}

\author{
I Sermet-Gaudelus, J C Souberbielle , I Azhar, J C Ruiz, P Magnine, V Colomb, C Le Bihan, D Folio, \\ G Lenoir
}

Arch Dis Child 2003;88:956-961

See end of article for authors' affiliations

......................

Correspondence to:

Dr I Sermet-Gaudelus,

Service de Pédiatrie

Générale, Hôpital Necker-

Enfants Malades, 149 rue

de Sèvres, 75015 Paris,

France; isabelle.sermet@

nck.ap-hop-paris.fr

Accepted 15 January 2003
Background: A major consequence of malnutrition in cystic fibrosis (CF) patients is the loss of lean body mass (LBM) and the subsequent impairment of respiratory muscle function.

Aim: To determine whether insulin-like growth factor I (IGF-I) could be related to the LBM depletion and the evolution of respiratory disease in CF patients.

Methods: LBM was evaluated by dual energy $x$ ray absorptiometry; serum concentrations of IGF-I were measured in 24 CF patients twice with a one year interval. Both values were expressed as SD score (SDS) calculated from normal data for age, sex, and pubertal stage and analysed with respect to anthropometric evaluation and disease related conditions.

Results: At the initial evaluation, IGF-I SDS had a mean value of -0.98 (range -3.6 to 3.2 ) and correlated with weight for age index, LBM SDS, and lung disease related conditions. Multiple regression analysis showed that only LBM remained independently related to IGF-I, suggesting that the relation of IGF-I to LBM was independent of weight and that the correlation between IGF-I and the respiratory conditions was related to the level of LBM. IGF-I SDS at the first evaluation was lower for the patients who lost $\geqslant 5 \%$ of weight for age index or $\geqslant 1$ SD of LBM between the two evaluations.

Conclusion: Low levels of IGF-I could be crucial for clinical outcome by impairing LBM and respiratory function. IGF-I could be a tool for nutritional evaluation by identifying the CF patients at risk of LBM depletion.
M alnutrition is an important prognostic factor in cystic fibrosis (CF) patients as it is associated with worsening of pulmonary status and poor survival. ${ }^{1}$ Failure to maintain normal body weight has been attributed to an energy intake that is inadequate to meet the increased energy expenditure due to the chronic bronchopulmonary infection. ${ }^{2}$ A major effect of malnutrition in patients with an already reduced fat body mass (FBM) is the loss of lean body mass (LBM). ${ }^{3}$ The associated wasting of respiratory muscles is an adverse prognostic factor because it adds to lung function degradation and increases morbidity. ${ }^{4}$ As an unapparent loss of skeletal muscle may occur in up to $25 \%$ of patients with a normal body weight, ${ }^{5}$ monitoring of LBM is important in evaluation of CF patients.

Insulin-like growth factor I (IGF-I) is an important circulating anabolic hormone promoting protein metabolism and inhibiting protein degradation. ${ }^{6}$ It also plays a critical role in myoblast proliferation and differentiation. ${ }^{7}$ Several studies have shown a diminished concentration of IGF-I in patients with $\mathrm{CF}$, and a relation between low body mass index (BMI) and declining IGF-I levels in CF patients. ${ }^{8-13}$ However, the clinical relevance of these low IGF-I levels still needs to be evaluated. We hypothesised that IGF-I may be causally related to the LBM depletion and disease severity in CF patients. To test this, we examined the relation between IGF-I concentrations and nutritional status, LBM, and disease related conditions in 24 CF patients.

\section{METHODS}

\section{Patients}

Twenty four patients attending the CF clinic for their annual assessment were recruited for this study. Mean age was 12.1 years (range 3.6-19.2). All the patients had exocrine pancreatic insufficiency and were taking replacement pancreatic enzyme therapy. Patients were required to be medically stable at the time of the study-that is, no important ongoing bronchial exacerbation and no hospital admission during the eight weeks preceding the study. Patients were excluded from participation if they had used oral or intravenous corticosteroids within six months of the study or if they had hepatopathy, glucose intolerance, or other endocrine disorders. Treatment with inhaled steroids was recorded.

The study was carried out in accordance with the Helsinki II declaration. As this was not an intervention study, there was no obligation to seek the approval of the local ethics committee.

\section{Evaluation}

The patients were seen by the same investigator at baseline (M0 evaluation) and one year later (M12 evaluation) between January 2000 and January 2002. Tanner stages (1-5) were used to determine pubertal stage. Pubertal stage classification was as followed at the first evaluation: ten P1, four P2, three P3, seven P5. Nutritional status was first assessed by anthropometric evaluation. Weight and height were recorded for each subject and measured under the same conditions. An electronic scale (Seca, Hamburg; precision $100 \mathrm{~g}$ ) was used for children weighing $>15 \mathrm{~kg}$. Height was measured using a Harpenden Stadiometer. Weight and

\footnotetext{
Abbreviations: $\mathrm{BMI}$, body mass index; $\mathrm{CF}$, cystic fibrosis; $\mathrm{DEXA}$, dual energy $x$ ray absorptiometry; $\mathrm{FBM}$, fat body mass; $\mathrm{FEV}_{1}$, forced expiratory volume in one second; FVC, forced vital capacity; IGF-I, insulin-like growth factor; IWA, ideal weight for age; IWH, ideal weight for height; LBM, lean body mass; RBP, retinol binding protein; $\mathrm{SaO}_{2}$, blood oxygen saturation; SDS, standard deviation score
} 
height were expressed as percent of ideal weight for age (\%IWA) and of ideal weight for height (\%IWH) in the statistical analysis instead of absolute values. Nutritional status was assessed according to the standards defined by the CF consensus report. ${ }^{14}$ Values were considered within normal range if \%IWH was $90-100 \%$, underweight if \%IWH was $85-$ $89 \%$, mild undernutrition if \%IWH was $80-84 \%$, moderate undernutrition if \%IWH was $75-79 \%$, and severe malnutrition if \%IWH was $<75 \%$. Malnutrition was thus defined as a \%IWH $<85 \%{ }^{14}$ Nutritional intake was estimated by the median of a three day diary energy intake and expressed as a percentage of the recommended dietary allowance for age. ${ }^{15}$

Body composition was evaluated by dual energy $x$ ray absorptiometry (DEXA) (Hologic QDR1000W/892 mef 1990, Hologic, Boston, MA) as described by Svendsen and colleagues. ${ }^{16}$ The LBM and FBM were expressed as standard deviation score (SDS) of reference values established with this apparatus for 50 control prepubertal children, 50 control adult men, and 50 control adult women. Mean (SD) LBM and FBM reference values were, respectively, for prepubertal children $79 \%(3 \%)$ and $18 \%(3 \%)$, for adult men $79 \%(3 \%)$ and $18 \%(3 \%)$, and for adult women $73 \%(3 \%)$ and $23 \%(3 \%)$ less than median ideal weight (personal data, Dr JC Ruiz). The precision for LBM and FBM measurement was $200 \mathrm{~g}$.

CF lung disease was assessed for a given patient by recording the number of antibiotic courses in the year preceding the study, and, at the M0 and M12 evaluation, by measuring the forced expiratory volume in one second $\left(\mathrm{FEV}_{1}\right)$, the forced vital capacity (FVC), and the blood oxygen saturation $\left(\mathrm{SaO}_{2}\right) . \mathrm{FEV}_{1}$ and FVC were expressed as percentages of normal predicted values for age and sex. Hypoxia was defined when $\mathrm{SaO}_{2}$ was $<95 \%$. The Shwachman score was calculated to assess overall disease severity at both evaluations. ${ }^{17}$ The maximum Shwachman score was 100 .

At the M0 and M12 evaluation, blood samples were taken for the evaluation of liver function, prealbumin, and retinol binding protein (RBP). Prealbumin and RBP reference ranges $(-2 \mathrm{SD}$ to $+2 \mathrm{SD})$ were respectively $0.2 \mathrm{l}-0.42 \mathrm{~g} / \mathrm{l}$ and $0.03-0.06 \mathrm{~g} / \mathrm{l}$.

\section{Assay}

Serum IGF-I was measured by immunoradiometric assay as previously described. ${ }^{18}$ The intra-assay coefficient of variation was $1.8 \%, 3.7 \%$, and $4.1 \%$ at $52 \mathrm{ng} / \mathrm{ml}, 307 \mathrm{ng} / \mathrm{ml}$, and $676 \mathrm{ng} / \mathrm{ml}$ respectively, and the inter-assay coefficient of variation was $5.8 \%, 4.5 \%$, and $5 \%$ at $56 \mathrm{ng} / \mathrm{ml}, 299 \mathrm{ng} / \mathrm{ml}$, and $700 \mathrm{ng} / \mathrm{ml}$ respectively. Results are expressed as the IGF-I SD score (IGF-I SDS) calculated from normal data for age, sex, and pubertal stage. Reference range was calculated using the local reference population. Data for prepubertal children $(\mathrm{n}=168)$ were previously published, ${ }^{18}$ whereas those for pubertal children are unpublished $(\mathrm{n}=100)$.

\section{Statistical design}

Statistical analysis was performed using the BMDP software package (University of California, Los Angeles). ${ }^{19}$ Data are presented as mean (SEM). Paired and unpaired data were respectively compared by the non-parametric Wilcoxon and Mann Whitney test. Correlation coefficients were calculated by simple regression analysis. Multiple stepwise regression analysis was used to clarify the most important determinants of the variance of LBM and IGF-I. The null hypothesis was rejected at $\mathrm{p}<0.05$.

\section{RESULTS}

\section{Patient characteristics}

Table 1 shows characteristics of the 24 patients included ( 10 boys, 14 girls) at entry and 12 months later. As there was no sex difference for these baseline parameters, all the following data will be presented with the sexes combined.

Eleven children presented with malnutrition. LBM SDS ranged from -5.3 to +2.3 . Sixteen patients had an LBM SDS lower than -2 . Among the 13 patients with a \%IWH $\geqslant 85 \%$, seven had an LBM SDS $\leqslant-2$. Ten patients had an FBM SDS $\leqslant-2$ (range -3 to +2.6 ). Use of inhaled steroids was not associated with malnutrition or abnormal body composition.

Fourteen patients had chronic Pseudomonas aeruginosa infection. Disease severity varied greatly as indicated by the range of FEV 1 (21-106) and FVC (23-120), the number of antibiotic courses for bronchial exacerbations in the year preceding the study (0-5), and the Shwachman score (40-100).

\section{LBM in relation to disease severity factors}

LBM SDS was correlated with weight expressed as \%IWA or IWH, but not with height (table 2). LBM SDS was also positively correlated with respiratory function and Shwachman score. It was inversely correlated with number of antibiotic courses in the year preceding the study. FBM SDS was also correlated with nutritional characteristics and disease related conditions (table 2). However, the relation between LBM and the disease severity was independent of FBM as shown by the following results. Using multiple regression analysis with $\mathrm{FEV}_{1}$ as the dependent variable and LBM SDS and FBM SDS as independent variables, only LBM SDS remained significantly associated (table 3). Similar results were found when FVC, Shwachman score, or the number of antibiotics were tested as the dependent variable (table 3).

\section{IGF-I in relation to nutritional status and body composition}

At entry, the mean value of IGF-I SDS was -0.98 (range -3.6 to 3.2$)$. Seventeen patients $(70 \%)$ had a serum level lower than 0 SD and eight (33\%) lower than -2 SD. The IGF-I SDS was correlated with weight expressed as \%IWA and $\%$ IWH but not with height (table 4). Sensitivity for malnutrition of IGF-I SDS $<0$ was 0.91 and specificity 0.33 . In contrast, a concentration of prealbumin and RBP $<-2$ SD had a sensitivity for malnutrition of 0.75 and 0.55 , respectively.

IGF-I was highly correlated with LBM (fig 1). IGF-I SDS levels were significantly lower in patients with an LBM SDS $<-2$ (table 5). All the patients with an LBM SDS $<-2$ had an IGF-I SDS $<-2$ (fig 1). In four cases, the IGF-I SDS was $<-2$ despite a \%IWH $\geqslant 85 \%$. However, in these four observations, LBM SDS was lower than -2 . IGF-I was also correlated with FBM. Using multiple regression analysis with LBM SDS, FBM SDS, and \%IWA as independent variables and IGF-I SDS as the dependent variable, only LBM SDS remained significantly associated with IGF-I SDS showing that neither weight or FBM are independently related to IGF-I after adjustment on LBM (table 3). This suggests that the relation of IGF-I to LBM is independent of weight and FBM.

\section{IGF-I in relation to disease severity factors}

The IGF-I SDS was correlated with FEV $_{1}$, FVC, Shwachman score, and number of antibiotic courses in the year preceding the study (table 4). All six patients with chronic hypoxia $\left(\mathrm{SaO}_{2}<95 \%\right)$ also had an IGF-I SDS $<-2$, but the association between hypoxia and IGF-I SDS did not reach significance. Using multiple regression analysis with $\mathrm{FEV}_{1}$ as the dependent variable and LBM SDS, FBM SDS, and IGF-I SDS in the equation, $\mathrm{FEV}_{1}$ was no longer correlated with IGF-I SDS and the only significant association was with LBM SDS $(F=9.69, p<0.01)$. The same results were found for FVC and Shwachman score. 
Table 1 Baseline characteristics of the 24 patients at inclusion (MO) and 12 months later (M12)

\begin{tabular}{llll}
\hline & MO & M12 & p value \\
\hline Age & $12.1(0.69)$ & $13.02(0.71)$ & $\mathrm{NA}$ \\
\%IWA & $81.9(3.2)$ & $81.3(3.3)$ & 0.3 \\
\%IWH & $89.4(2.43)$ & $90.2(2.78)$ & 0.45 \\
LBM SDS & $-2.81(0.48)$ & $-2.85(0.5)$ & 0.97 \\
FBM SDS & $-1.16(0.33)$ & $-1.49(0.49)$ & 0.42 \\
Shwachman score & $67.9(4.1)$ & $65.4(3.96)$ & 0.8 \\
FVC & $69(5.1)$ & $71.1(5.3)$ & 0.72 \\
FEV ${ }^{*}$ & $60.5(5.4)$ & $58.1(6)$ & 0.35 \\
Number of antibiotic courses in the year & $2.6(0.24)$ & $3.2(0.39)$ & 0.38 \\
preceding the study & $95.8(2.3)$ & $95.2(3.1)$ & 0.8 \\
SaO ${ }_{2}$ & $0.208(0.019)$ & $0.208(0.015)$ & 0.72 \\
Preallbumin & $0.046(0.013)$ & $0.029(0.002)$ & 0.48 \\
RBP & $-1.19(0.4)$ & $-1.16(0.4)$ & 0.87 \\
IGF-I SDS & $112(5.52)$ & $115(0.33)$ & 0.7 \\
Energy intake* & & \\
\hline Results are expressed as mean (SEM) and compared using the Wilcoxon test. & \\
\%IWA, percentage of ideal body weight for age; \%IWH, percentage of ideal body weight for height; LBM, lean \\
body mass; FBM, fat body mass; RBP, retinol binding protein; NA, not attributable. \\
*\% of predicted value for age and sex. \\
**\% of the recommended dietary allowance.
\end{tabular}

In order to test whether the relation between IGF-I and the disease related conditions was mediated by LBM, we compared the multiple correlation coefficients between IGF-I SDS and LBM SDS obtained in the two following nested multiple regression models: IGF-I SDS as the dependent variable and, in the equation, LBM SDS in the first model, and LBM SDS, $\mathrm{FEV}_{1}, \mathrm{FVC}$, Shwachman score, and number of antibiotic courses in the second model. The non-significant result $(\mathrm{F}=0.18$, NS) showed that the relation between IGF-I and the disease severity parameters did not exist any more after adjustment on LBM (table 3). This suggests that the relation between IGF-I and the disease severity parameters is related to the level of LBM.

\section{Evolution of clinical and anthropometric parameters, and IGF-I at one year interval}

At the end of the one year study, four patients had lost more than 5\% of \%IWA and six more than 0.5 SD of their LBM. The change in LBM accounted for $37 \%$ of the variation in weight. The mean change in IGF-I was not significant (table 1). All the patients with an IGF1 SDS $<0$ and $6 / 8$ of those with an IGFI SDS $<-2$ at the baseline evaluation remained in the same range at the end of the one year study. The correlations found at the baseline evaluation in univariate and

Table 2 Correlation between LBM SDS and FBM SDS and respiratory or nutritional parameters observed at the initial evaluation

\begin{tabular}{lll}
\hline Variable & $\begin{array}{l}\text { LBM SDS } \\
\boldsymbol{r} \text { ( } \mathbf{p} \text { value) }\end{array}$ & $\begin{array}{l}\text { FBM SDS } \\
\boldsymbol{r} \text { (p value) }\end{array}$ \\
\hline \%IWA & $0.79(<0.001)$ & $0.51(<0.01)$ \\
\%IWH & $0.92(<0.001)$ & $0.71(<0.001)$ \\
Height SDS & $0.21(0.33)$ & $-0.05(0.802)$ \\
FBM SD score & $0.4(0.05)$ & NA \\
Shwachman score & $0.62(0.002)$ & $0.41(0.06)$ \\
FVC & $0.65(<0.001)$ & $0.26(0.236)$ \\
FEV & $0.64(0.001)$ & $0.39(0.07)$ \\
Number of antibiotic courses & $-0.53(0.01)$ & $-0.29(0.19)$ \\
in the year preceding the study & & \\
Prealbumin & $0.52(0.03)$ & $-0.35(0.161)$ \\
RBP & $0.38(0.101)$ & $0.51(0.564)$ \\
IGF-I SDS & $0.63(0.001)$ & $0.53(0.01)$ \\
Energy intake & $0.38(0.06)$ & $0.29(0.17)$ \\
\hline
\end{tabular}

Results are presented as correlation coefficient and $p$ value. Abbreviations as for table 1 . multivariate analyses did not vary significantly at the M12 evaluation.

The change in weight between the baseline and the end of the study was correlated with IGF-I SDS at baseline $(r=0.48, \mathrm{p}=0.03)$ (fig 2$)$ but not with IGF-I change between the two evaluations (data not shown). The levels of IGF-I SDS at baseline were significantly lower for patients who lost $\geqslant 5 \%$ IWA between the two evaluations $(-3.07$ $(0.28)$ versus $-1.18(0.38) ; p=0.02)$. All patients who lost more than 1 SD of LBM also had a lower IGF-I SDS at the first evaluation, but this did not reach significance $(-1.88$ (0.41) versus $-0.97(0.76), p=0.28)$.

\section{DISCUSSION}

This prospective study shows that IGF-I is closely related to nutritional status in CF patients. Our data show a strong correlation between IGF-I, LBM, and disease severity. Since the levels of IGF-I were significantly decreased in patients with normal weight but low LBM, these results also suggest that low serum IGF-I could detect the patients with an isolated LBM depletion.

Similarly to previous observations, our results show a close correlation between IGF-I level and nutritional status in CF patients. ${ }^{8-13}$ This relation is mediated by the chronic pulmonary infection as suggested by the negative correlation of IGF-I with the number of antibiotic courses. This is in agreement with clinical reports showing normalisation of IGF-I after a short term course of intensive antibiotic therapy in CF patients ${ }^{13}$ and experimental data showing that IGF-I hepatic release is reduced during infection. ${ }^{20}$ In contrast to other studies, our results do not show a relation with the energy intake ${ }^{21}$ or chronic hypoxia, ${ }^{22}$ probably because of the small number of patients.

IGF-I appears to be a more reliable and sensitive indicator for malnutrition than prealbumin and RBP. Other studies have also reported low levels of IGF-I that contrast with normal concentrations of prealbumin and RBP in malnourished CF patients. ${ }^{23}{ }^{24}$ This superiority of IGF-I is supported by its prompt and marked increase during the early phase of nutritional repletion compared to the minimal changes of prealbumin and RBP. ${ }^{25}$ The reasons for this early increase are its closer nutrient dependency, ${ }^{26}$ its shorter half life, and its nycthemeral stability. ${ }^{6}$

IGF-I is correlated with LBM in our study. Most interestingly, this relation seems to be independent of weight and FBM, as shown by the independent relation between IGF-I 


\begin{tabular}{|c|c|c|c|c|}
\hline Model & $\beta$ & SE ( $\beta)$ & $\mathbf{F}$ & $\mathrm{p}$ value \\
\hline $\mathrm{FEV}_{1} \times \mathrm{LBM} \mathrm{SDS}^{2}$ & 4.69 & 1.51 & 9.66 & $<0.01$ \\
\hline $\mathrm{FEV}_{1} \times \mathrm{FBM} S \mathrm{SDS}$ & 2.32 & 2.89 & 0.64 & NS \\
\hline CVF $\times$ LBM SDS & 4.86 & 1.37 & 12.59 & $<0.01$ \\
\hline CVF $\times$ FBM SDS & -0.23 & 2.63 & 0.01 & NS \\
\hline Shwachman score $\times$ LBM SDS & 3.40 & 1.19 & 8.16 & 0.01 \\
\hline Shwachman score $\times$ FBM SDS & 2.28 & 2.28 & 1 & NS \\
\hline No. of antibiotic courses $\times$ LBM SDS & 3.25 & 1.39 & 5.47 & $<0.05$ \\
\hline No. of antibiotic courses $\times$ FBM SDS & 0.74 & 2.75 & 0.07 & NS \\
\hline IGF-I SDS $\times$ LBM SDS & 0.375 & 0.1 & 13.9 & $<0.01$ \\
\hline IGF-I SDS $\times$ LBM SDS & 0.36 & 0.15 & 5.26 & $<0.05$ \\
\hline IGF-I SDS $\times$ FBM SDS & 0.30 & 0.23 & 1.64 & NS \\
\hline IGF-I SDS × \%IWA & 0 & 0 & 1 & NS \\
\hline IGF-I SDS $\times$ LBM SDS & 0.35 & 0.14 & 6.42 & $<0.025$ \\
\hline IGF-I SDS $\times \mathrm{FEV}_{1}$ & 0 & 0.03 & & NS \\
\hline IGF-I SDS $\times$ FVC & -0.01 & 0.03 & 0.24 & NS \\
\hline IGF-I SDS $\times$ Shwachman score & -0.04 & 0.04 & 0.86 & NS \\
\hline IGF-I SDS $\times$ no. antibiotic courses & 0.08 & 0.03 & 4 & NS \\
\hline
\end{tabular}

and LBM in a multiple regression analysis with weight, FBM, and LBM tested as independent variables. This suggests that IGF-I could be a marker of selective LBM depletion. This detection is all the more important since low LBM can occur in CF patients with normal weight and is therefore often underestimated. Indeed, $54 \%$ of the patients with a \%IWH $\geqslant 85 \%$ had a LBM $\leqslant-2$ SD in our study; Abdullah et al also found hidden loss of skeletal muscle mass in one third of patients with a normal BMI. ${ }^{27}$

LBM depletion is a frequent characteristic of the nutritional status of CF patients. ${ }^{342728}$ One explanation is that nitrogen deposition is altered because of the consistent catabolic response associated with pulmonary infection. ${ }^{3} 29$ Moreover, in patients with an already reduced fat mass, the insufficient energy intake to counteract the increased energy needs leads to a negative energy balance with consumption of alternative substrates such as skeletal muscle. ${ }^{30}$ This particularly concerns respiratory muscles with reduction of inspiratory muscle mass and impairment of their function, as assessed by the correlation between reduction of muscle area and decreased sustained inspiratory muscle contraction already shown in clinical studies. ${ }^{41}{ }^{32}$ LBM depletion could therefore add to the severity of the lung disease, generating a

Table 4 Correlation between IGF-I SDS and characteristics of the patients observed at the initial evaluation

\begin{tabular}{ll}
\hline Variable & $r$ (p value) \\
\hline \%IWA & $0.52(0.01)$ \\
\%IWH & $0.67(<0.001)$ \\
Height SDS & $0.03(0.89)$ \\
LBM SDS & $0.63(0.001)$ \\
FBM SDS & $0.53(0.01)$ \\
Shwachman score & $0.52(0.01)$ \\
FVC & $0.44(0.039)$ \\
FEV 1 & $0.46(0.03)$ \\
Number of antibiotic courses in the year & $-0.62(0.003)$ \\
preceding the study & \\
Prealbumin & $0.51(0.036)$ \\
RBP & $0.49(0.03)$ \\
Energy intake & $0.19(0.22)$ \\
\hline
\end{tabular}

Results are presented as correlation coefficient and $p$ value. Abbreviations as for table 1 . vicious circle of poor bronchial drainage, increased work of breathing, greater number of exacerbations of pulmonary infection, and progressive pulmonary injury. ${ }^{3}$ This hypothesis is strongly supported by the correlation between the degree of pulmonary function impairment and LBM depletion reported in our results as in numerous studies. ${ }^{3} 2732$

IGF-I could play an important role in the maintenance of LBM via its anabolic properties, namely the stimulation of protein synthesis and accrual in skeletal muscle, and the delay of protein breakdown. ${ }^{73}$ This is supported by the increase in muscle mass in humans in response to administration of IGF- $\mathrm{I}^{34}$ and a study in patients undergoing continuous ambulatory peritoneal dialysis showing that the changes of LBM were correlated with those of IGF-I. ${ }^{35}$ IGF-I could also have a direct role at the cellular level because it is produced locally in skeletal muscle where it exerts a critical role in myoblast proliferation and terminal differentiation into postmitotic myotubes. ${ }^{36}$ Both general and local action

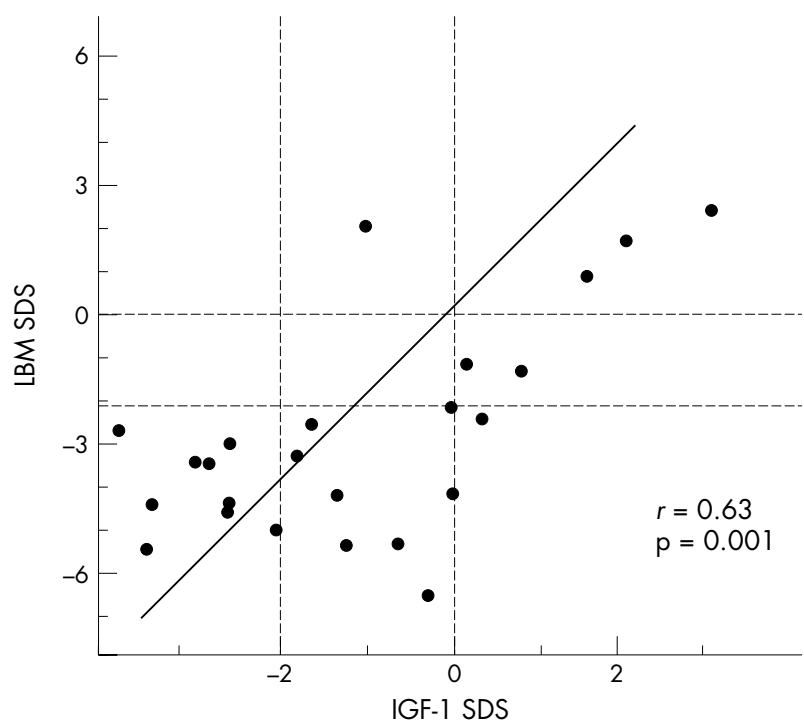

Figure 1 Relation between LBM SDS and IGF-I SDS observed at the initial evaluation. 
Table 5 Mean (SEM) IGF-I SDS level at TO according to nutritional status

\begin{tabular}{llll}
\hline $\begin{array}{l}\text { Nutritional characteristics } \\
\text { at TO }\end{array}$ & $\begin{array}{l}\text { Yes } \\
\text { Mean (SEM) }\end{array}$ & $\begin{array}{l}\text { No } \\
\text { Mean (SEM) }\end{array}$ & p value \\
\hline \%IWH $<0.85$ & $-1.46(0.37)$ & $-0.54(0.61)$ & 0.35 \\
LBM SDS $<-2$ & $n=11$ & $n=13$ & \\
FBM SDS $<-2$ & $-1.76(0.29)$ & $0.81(0.62)$ & 0.002 \\
& $n=16$ & $n=8$ & \\
& $-1.48(0.52)$ & $-0.84(0.45)$ & 0.7 \\
& $n=6$ & $n=18$ & \\
\hline
\end{tabular}

Comparison by Mann Whitney test. Abbreviations as for table 1 .

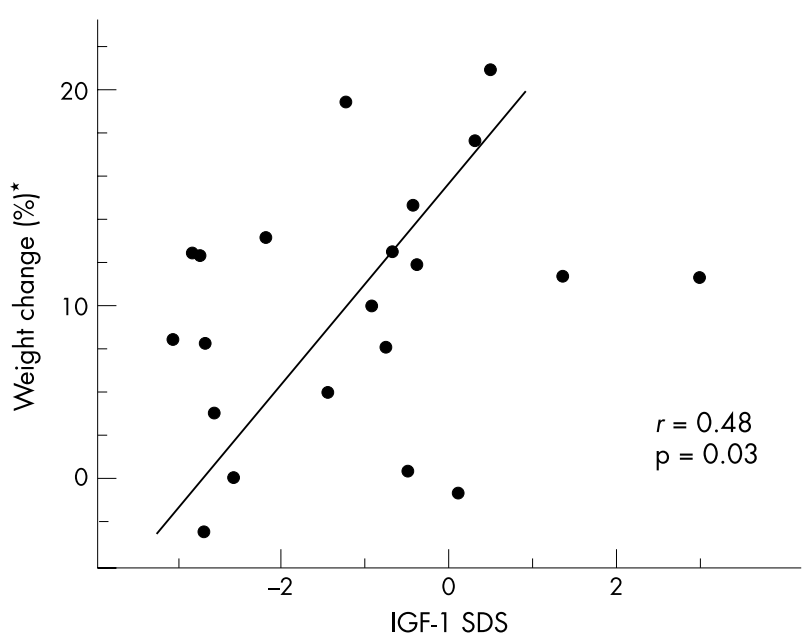

Figure 2 Relation between the variation in weight at one year interval and IGF-I SDS at the initial evaluation.

also involve the main respiratory muscle, the diaphragm, as suggested by animal studies showing that treatment with IGF-I prevents diaphragm fibre atrophy induced by moderate malnutrition and preserves its force generating capacity. ${ }^{37}$ IGF-I may thus be causally related to the evolution of CF pulmonary disease by hindering the loss of LBM and therefore maintaining respiratory function. Our finding that the correlation between IGF-I and pulmonary function is related to the level of LBM strongly supports this hypothesis.

The reduced IGF-I concentration in CF may therefore not only reflect but also contribute to the overall nutritional status. This is supported by the correlation between the IGF-I SDS at the beginning of the study and the variation in weight over the year of the study. We did not find a correlation between the changes in weight or LBM and those of IGF-I during the study. This however does not preclude the use of IGF-I as an indicator for nutritional outcome. First, low levels of IGF-I may result from the action of various factors such as infection or dietary intake that may change rapidly over the time. Second, the variations in IGF-I serum concentration occur relatively promptly, within about four days, as assessed by the very sensitive modifications of IGF-I level during short term changes in weight during normal growth ${ }^{38}$ or nutritional support. ${ }^{25}$ This suggests that relative changes in IGF-I are probably more useful than absolute values as indicators of short term changes in nutritional status and LBM acquisition. $^{39}$ Serial estimations of IGF-I in clinical practice may provide information on the nutritional risk and the disease activity. Detecting lowering of IGF-I could identify patients at risk of LBM depletion who need to be targeted for more intensive medical support. Conversely, detection of a rise in IGF-I is meaningful because it correlates with positive nitrogen balance and LBM acquisition. IGF-I could therefore be a useful tool for routine nutritional evaluation of $\mathrm{CF}$ patients.

In conclusion, this study provides evidence of a link between IGF-I, LBM maintenance, and lung disease in CF patients. IGF-I could play a crucial role in the evolution of CF via the following mechanism: starvation and catabolic response due to the vicious circle of pulmonary infection and decreased IGF-I serum levels. This leads to the loss of LBM that feeds back negatively on inspiratory muscle mass and function, adding to the problems of pulmonary impairment. We conclude that IGF-I is a predisposing factor for maintaining LBM in CF patients. Whether treatment with IGF-I could offer clinical efficacy and prevent LBM depletion by protecting against the catabolic effects of poor nutrition and infection, deserves further studies.

\section{Authors' affiliations}

I Sermet-Gaudelus, I Azhar, P Magnine, D Folio, G Lenoir, Service de Pédiatrie Générale, Hôpital Necker-Enfants Malades, 149 rue de Sèvres, Paris 75015, France

J C Souberbielle, Service d'Explorations Fonctionnelles, Hôpital NeckerEnfants Malades, 149 rue de Sèvres, Paris 75015, France

J C Ruiz, Service de Rhumatologie, Hôpital Cochin, 72 rue du Faubourg Saint Jacques, Paris 75014, France

V Colomb, Service de Gastro-Entérologie Pédiatrique, Hôpital NeckerEnfants Malades, 149 rue de Sèvres, Paris 75015, France

C Le Bihan, Service de Biostatistiques, Hôpital Necker-Enfants Malades, 149 rue de Sèvres, Paris 75015, France

\section{REFERENCES}

1 Corey M, McLaughlin FJ, Williams M, et al. A comparison of survival, growth and pulmonary function in patients with $\mathrm{CF}$ in Boston and Toronto. $J$ Clin Epidemiol 1988:41:583-91.

2 Reilly JJ, Edwards CA, Weaver LT. Malnutrition in children with cystic fibrosis: the energy-balance equation. J Pediatr Gastroenterol Nutr 1997;25:127-36.

3 lonescu AA, Nixon LS, Evans WD, et al. Bone density, body composition, and inflammatory status in cystic fibrosis. Am J Respir Crit Care Med 2000;162:789-94.

4 lonescu AA, Chatham K, Davies CA, et al. Inspiratory muscle function and body composition in cystic fibrosis. Am J Respir Crit Care Med 1998;158:1271-6.

5 Shoup R, Dalsky G, Warner S, et al. Body composition and health-related quality of life in patients with obstructive airways disease. Eur Respir $J$ 1997; 10:1576-80.

6 Thissen JP, Ketelslegers JM, Underwood LE. Nutritional regulation of the insulin-like growth factors. Endocrine Reviews 1994;15:80-101.

7 Singleton JR, Feldman EL. Insulin-like growth factor-I in muscle metabolism and myotherapies. Neurobiol Dis 2001;8:541-54.

8 Laursen EM, Juul A, Lanng S, et al. Diminished concentrations of insulin-like growth factor I in cystic fibrosis. Arch Dis Child 1995;72:494-7.

9 Taylor AM, Bush A, Thomson A, et al. Relation between insulin-like growth factor-l, body mass index, and clinical status in cystic fibrosis. Arch Dis Child 1997;76:304-9.

10 Arumugam R, Leblanc A, Seilheimer DK, et al. Serum leptin and IGF-I levels in cystic fibrosis. Endocrine Research 1998;24:247-57.

11 Taylor AM, Thomson A, Bruce-Morgan C, et al. The relationship between insulin, IGF-I and weight gain in cystic fibrosis. Clin Endocrinol 1999;51:659-65.

12 Laursen EM, Rasmussen MH, Koch C, et al. Normal spontaneous and stimulated GH levels despite decreased IGF-I concentrations in cystic fibrosis patients. Eur J Endocrinol 1999;140:315-21.

13 Lebl J, Zahradnikova M, Bartosova J, et al. Insulin-like growth factor-binding protein-3 in cystic fibrosis: a positive effect of antibiotic therapy and hyperalimentation. Acta Paediatr 2001;90:868-72.

14 Ramsey BW, Farrell PM, Pencharz P, et al. Nutritional assessment and management in cystic fibrosis: a consensus report. Am J Clin Nutr 1992;55:108-16.

15 Michel SH, Mueller DH. Practical approaches to nutrition care of patients with cystic fibrosis. Top Clin Nutr 1989;4:46-55.

16 Svendsen OL, Haarbo J, Hassager C, et al. Accuracy of measurements of body composition by dual-energy $\mathrm{x}$-ray absorptiometry in vivo. Am J Clin Nutr 1993;57:605-8

17 Shwachman H, Kulczycki LL. Long term study of one hundred five patients with cystic fibrosis. Am J Dis Child 1958;96:6-15.

18 Bussieres L, Souberbielle JC, Pinto G, et al. The use of insulin-like growth factor I reference values for the diagnosis of growth hormone deficiency in prepubertal children. Clin Endocrinol 2000;52:735-9.

19 Dixon WJ. BMDP statistical software manual. Berkeley: University Press of California, 1990.

20 Lang CH, Frost RA, Ejiofor J, et al. Hepatic production and intestinal uptake of IGF-1: response to infection. Am J Physiol 1998;275:G1291-8. 
21 Smith WJ, Underwood LE, Clemmons DR. Effects of caloric or protein restriction on insulin growth factor-I (IGF-I) and IGF-binding proteins in children and adults. J Clin Endocrinol Metab 1995:80:443-9.

22 Ross RJM, Miell JP, Freeman E, et al. Critically ill patients have high basal growth hormone levels with attenuated oscillatory activity associated with low levels of insulin-like growth factor-1. Clin Endocrinol 1991;35:47-54.

23 Benabdeslam H, Garcia I, Bellon G, et al. Biochemical assessment of the nutritional status of cystic fibrosis patients treated with pancreatic enzyme extracts. Am J Clin Nutr 1998;67:912-18.

24 Laaban JP, Kouchakji B, Dore MF, et al. Nutritional status of patients with chronic obstructive pulmonary disease and acute respiratory failure. Chest 1993; 103:1362-8

25 Clemmons DR, Underwood LE, Dickerson RN, et al. Use of plasma somatomedin-C/insulin-like growth factor-measurements to monitor the response to nutritional repletion in malnourished patients. Am J Clin Nutr 1985:41:191-8.

26 Caregero L, Favaro A, Santonastaso $P$, et al. Insulin-like growth factor 1 (IGF-1), a nutritional marker in patients with eating disorders. Clin Nutr 2001;20:251-7.

27 Abdullah EA, lonescu AA, Nixon LS, et al. Inflammatory response and body composition in chronic obstructive pulmonary disease. Am J Respir Crit Care Med 2001;164:1414-18.

28 lonescu AA, Nixon LS, Luzio S, et al. Pulmonary function, body composition, and protein catabolism in adults with cystic fibrosis. Am J Respir Crit Care 2002; 165:495-500

29 Baur LA, Waters DL, Allen BJ, et al. Nitrogen deposition in malnourished children with cystic fibrosis. Am J Clin Nutr 1991;53:503-11.
30 Bell SC, Bowerman AM, Nixon LS, et al. Metabolic and inflammatory responses to pulmonary exacerbation in adults with cystic fibrosis. Eur J Clin Invest 2000;3:553-9

31 Arora NS, Rochester DF. Effect of body weight and muscularity on human diaphragm mass, thickness and area. J Appl Physiol 1982;52:64-70.

32 Hayot M, Guillaumont S, Ramonatxo M, et al. Determinants of the tension time index of inspiratory muscles in children with cystic fibrosis. Pediatr Pulmonol 1997;23:336-43.

33 Thissen JP, Underwood LE, Ketelslegers JM. Regulation of insulin-like growth factor-1 in starvation and injury. Nutr Rev 1999:57:167-76.

34 Clemmons DR, Underwood LE. Role of insulin-like growth factors and growth hormone in reversing catabolic states. Horm Res 1992;38:37-40.

35 Numata M, Yamamoto H, Kawaguchi $Y$, et al. A study of association between lean body mass and serum insulin-like growth factor- 1 in continuous ambulatory peritoneal dialysis patients. Nippon Jinzo Gakkai Shi 1999;41:8-13.

36 Coolican SA, Samuel DS, Ewton DZ, et al. The mitogenic and myogenic actions of insulin-like growth factors utilize distinct signaling pathways. J Biol Chem 1997:272:6653-62.

37 Lewis MI, Feinberg AT, Fournier M. IGF-1 and/or growth hormone preserve diaphragm fiber size with moderate malnutrition. J Appl Physiol 1998;85: 189-97.

38 Gelander L, Blum WF, Larsson L, et al. Monthly measurements of insulin-like growth factor 1 (IGF-1) and IGF-binding protein-3 in healthy prepubertal children: characterization and relationship with growth: the 1-year growth study. Pediatr Res 1999;45:377-83.

39 Donahue SP, Philips LS. Response of IGF-I to nutritional support in malnourished hospital patients: a possible indicator of short-term changes in nutritional status. Am J Clin Nutr 1989:50:962-9.

\section{IMAGES IN PAEDIATRICS}

\section{Pseudoporphyria secondary to non-steroidal anti-inflammatory drugs}

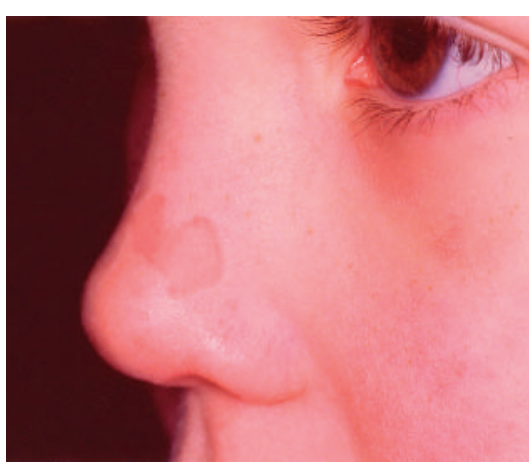

A 3 year old girl with juvenile idiopathic arthritis (JIA) presented with recurrent blisters over the cheeks and nose which were initially treated as impetigo with flucloxacillin. Despite this, new blisters continued to appear which evolved into punched out lesions and healed with scars. She had been treated with naproxen $125 \mathrm{mg} /$ day for six months. This is pseudoporphyria, an uncommon but well recognised side effect of non-steroidal antiinflammatory drugs (NSAIDs), with cutaneous features in photosensitive areas indistinguishable from porphyria, but without the metabolic porphyrin abnormalities. ${ }^{1}$ The incidence of pseudoporphyria may be as high as $10 \%$ in children taking NSAIDs for JIA. ${ }^{12}$ Naproxen is the most common culprit, but the condition has been reported with other NSAIDs. ${ }^{3-7}$ Stopping the naproxen ceased the new blister formation and the ulcers healed, but faint scars remain. It is important for paediatricians to be aware of this disfiguring side effect of frequently prescribed drugs, as discontinuing the drug produces resolution. ${ }^{8}$

P Bryant, P Lachman Northwick Park Hospital penelope.bryant@doctors.org.uk

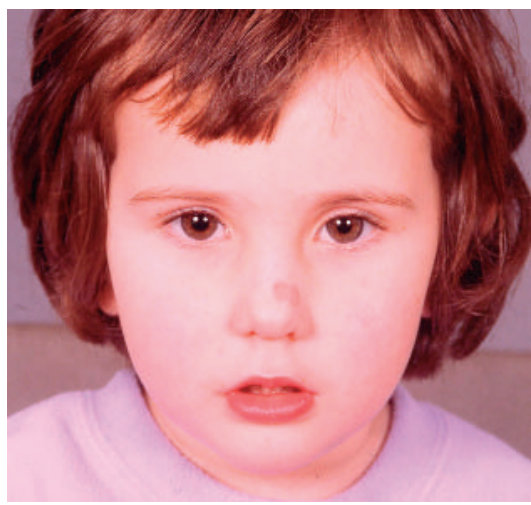

\section{References}

1 De Silva B, Banney L, Uttley W, et al. Pseudoporphyria and nonsteroidal antiinflammatory agents in children with juvenile idiopathic arthritis. Pediatr Dermatol 2000;17:480-3.

2 Wallace CA, Farrow D, Sherry DD. Increased risk of facial scars in children taking nonsteroidal antiinflammatory drugs. J Pediatr 1994;125(5 pt 1):819-22.

3 Creemers MC, Chang A, Franssen MJ, et al. Pseudoporphyria due to naproxen. A cluster of 3 cases. Scand J Rheumatol 1995;24:185-7.

4 Cheketts SR, Morrison KA, Baughman RD. Nonsteroidal anti-inflammatory-induced pseudoporphyria: is there an alternative drug? Cutis 1999:63:223-5.

5 Al-Khenaizan S, Schechter JF, Sasseville D. Pseudoporphyria induced by propionic acid derivatives. J Cutan Med Surg 1999;3:162-6.

6 Krischer J, Scolari F, Kondo-Oestreicher M, et al. Pseudoporphyria induced by nabumetone. J Am Acad Dermatol 1999:40:492-3.

7 O'Hagan AH, Irvine AD, Allen GE, et al. Pseudoporphyria induced by mefenamic acid. Br J Dermatol 1998;139:1131-2.

8 Anon. Drug-induced cutaneous photosensitivity: some drugs warrant routine precautions. Prescrire Int 2000;9(48): 117-22. 